\section{OPEN ACCESS}

Edited by:

Błazej Misiak,

Wroclaw Medical University, Poland

Reviewed by:

Keshen Li,

Jinan University, China

Adonis Sfera,

Loma Linda University, United States

*Correspondence:

Napoleon Waszkiewicz

napoleonwas@yahoo.com

Specialty section

This article was submitted to

Schizophrenia

a section of the journal

Frontiers in Psychiatry

Received: 25 March 2018

Accepted: 15 June 2018

Published: 05 July 2018

Citation:

Kandratsenka H, Nestsiarovich $A$,

Goloenko I, Danilenko N,

Makarevich A, Obyedkov V, Davydenko O and Waszkiewicz N (2018) Association of MIR137 With

Symptom Severity and Cognitive

Functioning in Belarusian

Schizophrenia Patients.

Front. Psychiatry 9:295.

doi: 10.3389/fpsyt.2018.00295

\title{
Association of MIR137 With Symptom Severity and Cognitive Functioning in Belarusian Schizophrenia Patients
}

\begin{abstract}
Hanna Kandratsenka ${ }^{1}$, Anastasiya Nestsiarovich ${ }^{2}$, Inna Goloenko ${ }^{1}$, Nina Danilenko ${ }^{1}$, Anna Makarevich ${ }^{1}$, Victor Obyedkov ${ }^{3}$, Oleg Davydenko ${ }^{1}$ and Napoleon Waszkiewicz ${ }^{4 *}$

1 Laboratory of Cytoplasmic Inheritance, Institute of Genetics and Cytology, National Academy of Sciences of the Republic of Belarus, Minsk, Belarus, ${ }^{2}$ Department of Internal Medicine, Center for Global Health, University of New Mexico, Albuquerque, NM, United States, ${ }^{3}$ Department of Psychiatry and Medical Psychology, Belarusian State Medical University, Minsk, Belarus, ${ }^{4}$ Department of Psychiatry, Medical University of Białystok, Białystok, Poland
\end{abstract}

MicroRNA-137 (miRNA-137; miR-137) is one of the important post-transcriptional regulators of the nervous system development, and its MIR137 gene rs1625579 polymorphism was reported to be a potential regulator for schizophrenia susceptibility. However, schizophrenia characteristics controlled by MIR137 rs1625579 polymorphism are still insufficiently understood. There were 3 groups included in the study: (a) subjects with diagnosis of schizophrenia ( $n=150$; 81-females, 69-males), (b) mentally healthy people (control group; $n=102$; 66-females, 36-males) and (c) Belarusian indigenous male group ( $n=295)$. Associations of rs1625579 with schizophrenia, symptom's severity and cognitive performance [by using Positive and Negative Syndrome Scale (PANSS) and Wisconsin Card Sorting Test (WCST), respectively] were studied, when compared to controls. Allele and genotype frequencies were investigated in Belarusian indigenous males. Rs1625579 displayed no association with schizophrenia in Belarusian population. Significant "symptom severity-genotype" interactions were revealed for schizophrenia patients. Patients with T/G genotype displayed lower severity of positive symptoms and general psychopathology compared to homozygous subjects. $\mathrm{T} / \mathrm{T}$ genotype was associated with the highest symptom's severity. The negative symptom scores and the total PANSS-score were significantly higher in females carrying genotype $T / T$ vs. $T / G+G / G$; no significant gene-phenotype associations were found in males. WCST parameters did not show any association with rs1625579 polymorphism. MIR137 rs1625579 polymorphism might be an important sex-dependent factor influencing severity of schizophrenia psychopathological manifestations. These findings also contribute to the knowledge on candidate gene effects on characteristics related to schizophrenia phenotype. As miR 137 is considered to be cancer therapeutic target, miR-137 may also explain the lower incidence of cancer in schizophrenia patients. Further studies with larger sample size are needed to confirm these novel findings. 


\section{INTRODUCTION}

According to the current concepts, mental disorders are the result of the complex interactions between genetic determinants and epigenetic factors. Molecular mechanisms of epigenetic modifications include histone modifications of chromatin, DNA methylation, RNA editing, as well as the effect of non-coding RNA molecules such as small interfering RNA (siRNA) and microRNA (miRNA) (1). MicroRNAs (miRNAs) are important post-transcriptional regulators of gene activity, the changes of its expression and functional status may have various consequences. MiRNAs were recently reported to be related to rheumatoid arthritis, systemic lupus erythematosus, diabetes, multiple sclerosis, asthma, psoriasis, inflammatory bowel diseases, dermatological disorders, psychiatric and neurological disorders (2). The aberrant expression of miRNAs was shown to be associated with schizophrenia, autism, Down syndrome, Rett syndrome, Fragile X syndrome, and depression (2, 3). A number of severe mental disorders, particularly schizophrenia, are characterized by presence of comorbid diseases. Such conditions may be partly explained by the variations in miRNAs functioning-one of the key elements of the gene set regulation that might be intrinsically related to the common etiology of several diseases.

The Genome-Wide Association Studies (GWAS) have shown that MIR137 gene may be considered as a strong candidate to be included to the existing list of 108 gene loci of schizophrenia susceptability (4-6). Some lines of evidence show that individuals suffering from mental disorders, in particular schizophrenia, displayed altered MIR137 expression as well as its target genes (6). MiRNA-137 is known to regulate the expression of ca. 1 900 other genes including several genes independently associated with schizophrenia (7). Such well-known candidate genes targeted by miR-137 include BDNF, ZNF804A, TCF4, CACNA1C, CSMD, and C10ORF26 $(8,9)$. The product of MIR137 gene, microRNA-137 (miR-137), may be involved in pathophysiology of schizophrenia by affecting synaptogenesis, neurogenesis in adults, synaptic plasticity as well as many other aspects of the central nervous system development $(6,9,10)$. The mice models confirmed the effect of miR-137 on neurogenesis, proliferation and maturation of the neural stem cells (11). The level of miR137 in adult brain tissue is about 100 times higher than in embryonic one, thus indicating that MIR137 gene expression increases during brain development and neuron differentiation (8). Several brain regions including subcortical (caudate nucleus, putamen), cortical (orbito-frontal cortex) areas and synaptodendritic neuron compartments are enriched with MIR137 (8).

Recently, special attention was drawn to the study of MIR137 association with the structural brain abnormalities and the patterns of functional activation of certain brain areas. Several lines of evidence displayed controversial results, one of them found that schizophrenia patients with T/T genotype of MIR137 rs1625579 polymorphism, but not healthy subjects, had abnormal size of subcortical structures (increased lateral ventricles and reduced hippocampus); other authors failed to confirm this association neither in patients nor in the healthy subjects $(8,12)$. Li and $\mathrm{Su}(13)$ reported the data on rs1625579 polymorphism association with the size of amygdala, caudate nucleus, hippocampus, shell and the total brain volume in 299 Chinese and 5775 Caucasian subjects. In Chinese population, significant associations were found for hippocampus size only, with smaller size observed in T allele carriers (13).

Currently, the studies of MIR137 rs1625579 association with susceptibility to schizophrenia hold the leading position, nevertheless the number of data points to the contradictory results for different ethnic groups (Table 1, Figure 1). GWAS studies have shown the association between $\operatorname{rs} 1625579$ ( $p=1.6$ $\times 10-11)$ SNP with schizophrenia, however, it is still difficult to explain the functional significance of the SNP localized in the intron region of MIR137 primary transcript $(6,12)$. The major T-allele of rs1625579 polymorphism was identified as a risk factor for schizophrenia. Postmortem studies showed that subjects with $\mathrm{T} / \mathrm{T}$ genotype have reduced level of MIR137 expression compared to other genotypes carriers that enabled to suggest the impact of this locus on MIR137 gene expression $(1,6,12)$.

British scientists made an attempt to clarify the functional significance of rs1625579 locus for MIR137 gene. It was shown that rs1625579 is in linkage disequilibrium with the rs2660304 polymorphism located within an internal promoter of (Imir137) MIR137 gene. By using the reporter gene construct it was showed that rs2660304 alleles affect transcriptional activity of the Imir137 promoter of MIR137 gene. As rs1625579 (a risk factor for schizophrenia), is in linkage disequilibrium with rs2660304, a possible functional role of rs1625579 as a factor regulating MIR137 expression by means of rs2660304 polymorphism was suggested (6).

The data pool regarding MIR137 effect on schizophrenia liability is increasing; however, the understanding of schizophrenia clinical features regulated by this gene is still insufficient. In the present publication, we analyzed the association of MIR137 rs1625579 gene polymorphism with susceptibility to schizophrenia in Belarusian population, as well as its relationship with symptom severity and cognitive functioning.

\section{MATERIALS AND METHODS}

\section{Object of the Study}

There were 3 groups (mean age \pm SD $[46.8 \pm 9.4]$, minmax [24-65]) included in the study: (a) subjects with diagnosis of schizophrenia according to ICD-10 criteria (main group), (b) mentally healthy people (control group), (c) Belarusian indigenous group. Schizophrenia patients consisted of 150 individuals (81 females and 69 males), control group consisted of 102 people (66 females and 36 males), and Belarusian indigenous group contained 295 males whose ancestors lived in Belarus for at least 3 generations. There were no age differences between groups. Recruitment of subjects with schizophrenia was carried out by psychiatrists of the Republican Research and Practice Center for Mental Health (RPCMH, Minsk) among the inpatients undergoing the course of treatment. The study was approved by the ethic committee of the RPCMH, all participants gave signed written informed consent and biological samples (capillary blood) were taken afterwards. 
TABLE 1 | Association studies of MIR137 rs1625579 polymorphism with risk of schizophrenia.

\begin{tabular}{|c|c|c|c|c|c|c|c|c|c|c|}
\hline \multirow[t]{3}{*}{ Source } & \multirow[t]{3}{*}{ Population } & \multicolumn{4}{|c|}{ Schizophrenia patients } & \multicolumn{4}{|c|}{ Healthy controls } & \multirow[t]{3}{*}{$P$-value } \\
\hline & & \multirow[t]{2}{*}{$N$} & \multicolumn{3}{|c|}{ Genotype frequencies (\%) } & \multirow[t]{2}{*}{$N$} & \multicolumn{3}{|c|}{ Genotype frequencies (\%) } & \\
\hline & & & $\mathbf{T} / \mathbf{T}$ & $\mathrm{G} / \mathrm{T}$ & G/G & & $\mathbf{T} / \mathbf{T}$ & $\mathrm{G} / \mathrm{T}$ & G/G & \\
\hline Sun et al. (14) & China & 589 & 86.9 & 12.2 & 0.9 & 622 & 86.8 & 12.7 & 0.5 & $p>0.05$ \\
\hline Kuswanto et al. (8) & Singapore & 84 & 89.2 & \multicolumn{2}{|c|}{10.8} & 63 & 88.9 & \multicolumn{2}{|c|}{11.1} & $p>0.05$ \\
\hline Yuan et al. (15) & China & 506 & 87.9 & 11.7 & 0.4 & 522 & 89.6 & 10.4 & 0 & $p>0.05$ \\
\hline Wang et al. (16) & China & 300 & 89.7 & 10.3 & 0 & 300 & 88.3 & 11.7 & 0 & $p>0.05$ \\
\hline Guan et al. (10) & China & 1429 & 77.7 & 20.8 & 1.5 & 1570 & 74 & 23.6 & 2.4 & $p=0.023$ \\
\hline Van Erp et al. (11) & US & 48 & 81.25 & 18.75 & 0 & 63 & 73.02 & 23.81 & 3.17 & $p>0.05$ \\
\hline \multirow[t]{3}{*}{ Hommers et al. (5) } & GWAS, 2014 & \multicolumn{4}{|c|}{$\begin{array}{l}\text { Caucasians } \\
\mathrm{n} \text { (patients) }=33.332 \\
\mathrm{n} \text { (controls) }=27.888\end{array}$} & & & & & $p<0.05$ \\
\hline & GWAS, 2013 & \multicolumn{4}{|c|}{$\begin{array}{l}\text { Sweden samples: } \\
\text { n (patients)=5.001, } \\
\text { n (controls)=6.243; } \\
\text { Repeated study } 1 \text { (Caucasians): } \\
\text { n (patients)=8.832, } \\
\text { n (controls)=12.067; } \\
\text { Repeated study } 2 \text { (Caucasians): } \\
\text { n (patients)=7.413, } \\
\text { n (controls) }=19.762\end{array}$} & & & & & $p<0.05$ \\
\hline & GWAS, 2011 & \multicolumn{4}{|c|}{$\begin{array}{l}\text { Caucasians } \\
\mathrm{n} \text { (patients) }=9.394 \\
\mathrm{n} \text { (controls)=12.462 } \\
\text { Repeated study } \\
\mathrm{n} \text { (patients) }=8.432 \\
\mathrm{n} \text { (controls) }=21.397\end{array}$} & & & & & $p<0.05$ \\
\hline
\end{tabular}

GWAS, genome wide association study. Bold values indicate the mean statistical significance or \% value.

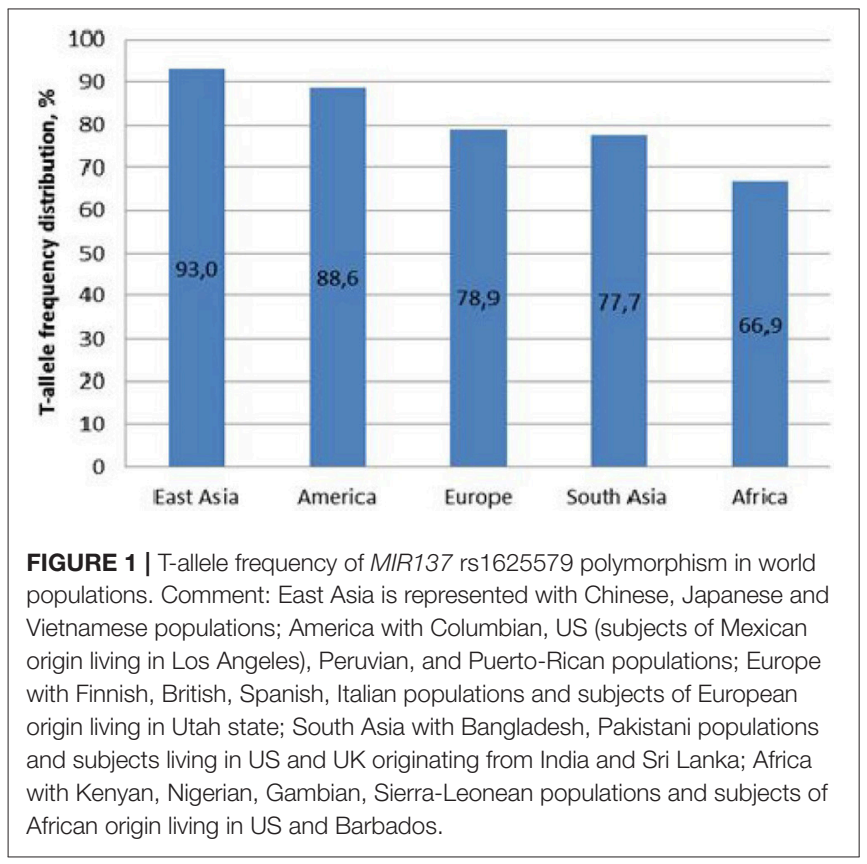

Clinical assessment of patients was performed by psychiatrists of RPCMH at the stage of schizophrenia "clinical outcome" which reflects the period of maximal symptoms consolidation and appears 12-15 years after the onset of psychotic symptoms (17). The following inclusion criteria were applied: age 18-65 years, disease duration over 12 years, no evidence of regular neuroleptics intake during 1-3 weeks prior to hospitalization, absence of any comorbid psychiatric disorders (except of nicotine dependence), absence of severe somatic states and neurological disturbances. The control group consisted of healthy individuals. These subjects were presented by volunteers of different sociodemographic groups (students, workers of medical and research institutions).

To increase the statistical power, a sample of Belarusian indigenous population containing 295 males whose ancestors lived in Belarus for at least 3 generations was included into this study. The biological material of the participants was obtained from the population DNA bank previously created in the Laboratory of Cytoplasmic Inheritance. Some details of all the samples are provided in Table 2.

\section{Clinical Measurements}

Patients were examined during the period of acute schizophrenia exacerbation ("relapse"). Majority of symptoms were measured during the first 3 days of hospitalization once a patient was able to establish the contact with investigator. To minimize the impact of the maintenance neuroleptic therapy on the disease course and relapse symptomatic structure, only patients who were not regularly taking antipsychotics during $1-3$ weeks prior to the current hospitalization were selected for examination. This 
information was obtained from patient's caregivers, as well as from medical documentation (including the referrals).

Symptom severity was assessed by means of the semistructured clinical interview and the psychometric scale PANSS (Positive and Negative Syndrome Scale) which consists of 33 items, rated from 1 to 7 scores each. All the items are arranged into four sub-scales reflecting the severity of positive symptoms, negative symptoms, general psychopathology and additional symptoms (18).

The cognitive functioning was evaluated by means of Wisconsin Card Sorting Test (WCST) - a widely used tool to assess the working memory, attention, abstract thinking and executive functioning (19). During the test it is presented a series of cards on the computer monitor with figures differing by either color, number or shape. The patient is asked to sort each of the 128 cards into four categories based on a single currently active criteria which changes from time to time. The test is considered to measure the ability of a testee to identify and follow the classification algorithm, and to productively "switch" once the rules are changed. We evaluated the following test variables: (1) perseverative errors which reflect the problems in switching from one sorting rule to another; (2) non-perseverative errors which are associated with absent-mindedness of attention; (3) total of all errors which reflects a functional status of cognitive flexibility and attention of the examinee; (4) total amount of categories completed which reflects the cognitive processing success during the test; (5) total amount of cards to complete the first category which reflects the efficiency/speed of identifying the first classification algorithm. The most informative variable for executive functioning is "perseverative errors."

\section{Genotyping}

Genomic DNA was extracted from peripheral blood leukocytes using the phenol-chloroform extraction (20). Genotyping of rs1625579 MIR137 was performed using TaqMan assay. The primers and probes used for PCR amplification were designed by the Beacon Designer 7.91. software (primers: forward-5'-CCCTAGTTGAAAATATTTC$3^{\prime}$ and revers-5'-GGGTCACTATTATTTAACA-3'/probes: FAM-5' -TGTTAATCACAATTACATCAACTCAG-3'; HEX5'-TGTTAATCACAATTAAATCAACTCAG-3' ${ }^{\prime}$. PCR amplification was performed in a $25-\mu 1$ reaction volume, which contained 2X Mix (Primetech, Minsk, Belarus: 0.04 $\mathrm{u} / \mu$ of Diamant FAST DNA polymerase $10 \mathrm{X}$ Buffer $\ll$ FAST $\gg$

TABLE 2 | Gender characteristic in patients and controls.

\begin{tabular}{lccc}
\hline Characters & Patients & Controls & $\mathbf{P}^{\mathbf{1}}$ \\
\hline $\mathbf{N}$ & 150 & 102 & - \\
Gender & & & 0.118 \\
Female & $54.0 \%$ & $64.7 \%$ & \\
Male & $46.0 \%$ & $35.3 \%$ &
\end{tabular}

$P^{1}$, Comparison between the patient group and control group using Chi-square method; $S D$, standard deviation. containing $4.5 \mathrm{mM} \mathrm{MgCl} 2,0.2 \mathrm{mM}$ of each dNTP), $0.3 \mathrm{pM}$ of each primer and probe, and 30-40 ng of genomic DNA. The conditions used for thermal cycling included initial denaturation at $95^{\circ} \mathrm{C}$ for $2 \mathrm{~min}, 10$ cycles without fluorescence detection at $95^{\circ} \mathrm{C}$ for $15 \mathrm{~s}$, at $58^{\circ} \mathrm{C}$ for $30 \mathrm{~s}$ and at $72^{\circ} \mathrm{C}$ for $30 \mathrm{~s}$, and a final elongation at $72^{\circ} \mathrm{C}$ for $40 \mathrm{~s}$ after that 35 cycles with the same mode but with fluorescence detection. The described thermal cycling was performed with the Real-Time Stratagene Mx 3005P (Agilent Technologies, Santa Clara, California). Allele designations for MIR137 rs1625579 are according to the NCBI reference sequences and the dbSNP database (https://www.ncbi. nlm.nih.gov/projects/SNP/snp_ref.cgi?rs=1625579).

\section{Statistical Analysis}

The association of the studied locus polymorphism with schizophrenia symptom severity and cognitive functioning was tested with SPSS software Version 22.0. The two-tailed estimation of significance used in the analysis was defined at $P<0.05$. The Hardy-Weinberg (HWE) equilibrium was verified for patients and controls. The presence of HWE was tested by chi-square test $\left(\chi^{2}\right)$ for goodness of fit. Chi-square method (Pearson's X2) was used to evaluate rs1625579 locus for the risk of schizophrenia. Association of rs1625579 variants with variables related to schizophrenia symptom severity and cognitive functioning was analyzed using non-parametric Kruskal-Wallis $H$-Test and Mann-Whitney $U$-test. Association of rs 1625579 polymorphism with symptom severity and sex was tested using two-way ANOVA.

\section{RESULTS}

Analysis of genotype and allele distribution of MIR137 rs1625579 polymorphism in both compared groups has shown the $\mathrm{T}$ allele prevalence and has not revealed any significant differences between patients and controls (Table 3). The distribution of Allele frequencies of the locus studied in the control group corresponded to that known for European populations ("1000 genomes" database) (Electronic source: http://browser. 1000genomes.org/Homo_sapiens/Variation/Population?db= core; $\mathrm{r}=1: 98502434-98503434 ; \mathrm{v}=\mathrm{rs} 1625579 ; \mathrm{vdb}=$ variation; $\mathrm{vf}=$ 1146801\#373401_tablePanel). The allele frequencies were consistent with Hardy-Weinberg equilibrium for patients and controls. Allele frequencies analysis in a sample of Belarusian indigenous population has demonstrated high $\mathrm{T}$ allele total frequency: $\mathrm{T}$ allele $-75.9 \%$; $\mathrm{G}$ allele $-24.1 \%$. The detailed data on the genotype frequencies in Belarusian indigenous population compared to the world-wide populations obtained within the "1000 genomes" project are presented in Table 4.

TABLE 3 | Allele frequencies distribution of MIR137 rs1625579 polymorphism among schizophrenia patients and healthy controls.

\begin{tabular}{lccc}
\hline Allele & Patient group & Control group & P-level; $\boldsymbol{\alpha}=\mathbf{0 . 0 5}$ \\
\hline $\mathrm{T}$ & $77 \%$ & $74 \%$ & $p=0.459$ \\
$\mathrm{G}$ & $23 \%$ & $26 \%$ &
\end{tabular}


A comparative analysis of the genotype frequencies and combinations ( $T / T$ vs. $T / G+G / G)$ of rs 1625579 polymorphism has not revealed any significant differences between schizophrenia patients and healthy controls (Table 5). The non-significant increase of $\mathrm{T} / \mathrm{T}$ genotype frequency was observed in patient group compared to the control group, and this trend persisted when sexual differences were taken into account (patient female $\mathrm{T} / \mathrm{T}-65.4 \%$ vs. control female $\mathrm{T} / \mathrm{T}-$ $54.5 \%$; patient male $\mathrm{T} / \mathrm{T}-55.1 \%$ vs. control male $\mathrm{T} / \mathrm{T}-50 \%$ ). However, the overall genotype frequencies distribution did not differ significantly between the comparison groups, both in males and females ( $p=0.480$ and $p=0.362$, respectively). Also, we didn't find any differences in allele and genotype distribution between male patients and a sample of Belarusian indigenous population $(p=0.579)$.

We analyzed MIR137 rs1625579 polymorphism association with the schizophrenia symptom severity using non-parametric Kruskal-Wallis-H-test for genotypes, and Mann-Whitney $U$ test for genotype combinations in 150 schizophrenia patients. The average scores for the different PANSS subscales (positive symptoms, negative symptoms, general psychopathology) and the general index for all subscales of psychometric PANSS are presented in Figure 2. These data demonstrate that rs1625579 polymorphism is significantly associated with positive symptoms and general psychopathology scores as well as with the total

TABLE 4 | Genotype distribution of MIR137 rs1625579 polymorphism in world populations.

\begin{tabular}{|c|c|c|c|c|c|c|}
\hline \multirow[t]{3}{*}{ Populations } & \multicolumn{6}{|c|}{ Genotypes } \\
\hline & \multicolumn{2}{|c|}{$T / T$} & \multicolumn{2}{|c|}{ T/G } & \multicolumn{2}{|c|}{ G/G } \\
\hline & $n$ & $\%$ & $n$ & $\%$ & $n$ & $\%$ \\
\hline $\begin{array}{l}\text { Belarusian indigenous } \\
\text { population, } n=295\end{array}$ & 178 & 60.3 & 92 & 31.2 & 25 & 8.5 \\
\hline East Asia, $n=504$ & 437 & 86.7 & 63 & 12.5 & 4 & 0.8 \\
\hline America, $n=347$ & 274 & 79.0 & 67 & 19.3 & 6 & 1.7 \\
\hline Europe, $n=503$ & 314 & 62.4 & 166 & 33.0 & 23 & 4.6 \\
\hline South Asia, $n=489$ & 288 & 58.9 & 184 & 37.6 & 17 & 3.5 \\
\hline Africa, $n=661$ & 306 & 46.3 & 273 & 41.3 & 82 & 12.4 \\
\hline
\end{tabular}

Structure of presented populations is identical to that in Figure 1. Bold values indicate the mean statistical significance or \% value. symptomatology score in patients with schizophrenia. To achieve a higher resolution of the observed trend, we analyzed the genotype and genotype combination associations with each of the symptom complex (Table 6). The results obtained show the presence of a "heterozygote effect" of rs1625579, which implies that in subjects possessing heterozygous TG genotype, the severity of symptom (PANSS_P, PANSS_G, PANSS_Total) is significantly lower when compared to homozygous subjects. At the same time, $\mathrm{T} / \mathrm{T}$ genotype is the most unfavorable for the abovementioned symptoms, syndromes and general psychopathological state of patients. It's worthy to note that statistical power of the studied sample was limited by a low number (10) of $G / G$ homozygous subjects. It caused the simultaneous presence of significant differences between $\mathrm{G}+$ ( $\mathrm{T} / \mathrm{G}+\mathrm{G} / \mathrm{G})$ and $\mathrm{G}-(\mathrm{T} / \mathrm{T})$ genotype combination (in this case G/G genotype plays a protective role) as well as between $\mathrm{T} / \mathrm{T}+\mathrm{G} / \mathrm{G}$ and $\mathrm{T} / \mathrm{G}$ genotype combinations (in this case $\mathrm{G} / \mathrm{G}$ genotype has a negative effect upon symptoms severity) for PANSS_P and PANSS_Total indices. That's why a definite statement on G/G genotype effect on schizophrenia symptom

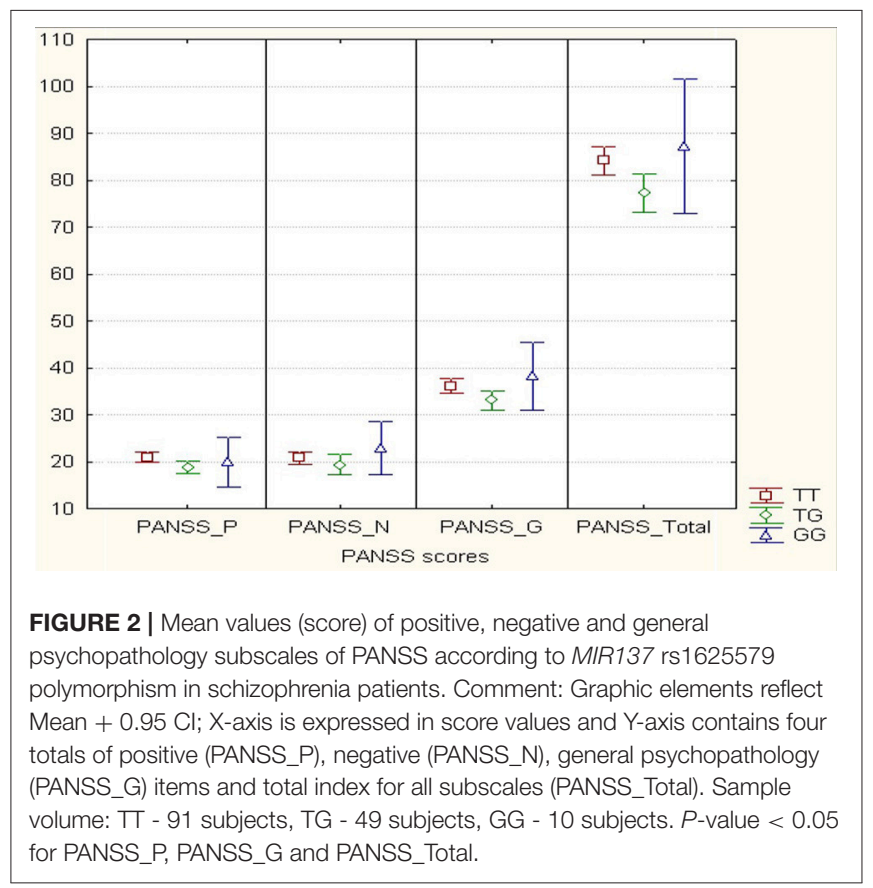

TABLE 5 | Genotype frequencies distribution of MIR137 rs1625579 polymorphism in schizophrenia patients and healthy controls.

\begin{tabular}{lccrc}
\hline Genotype & Patient group, \% (subjects) & Control group, \% (subjects) & $\begin{array}{c}\boldsymbol{P} \text {-level; } \alpha=\mathbf{0 . 0 5} \\
\text { (95\% Cl) }\end{array}$ \\
\hline T/T & $60.7 \%(91)$ & $52.9 \%(54)$ & 0.244 & $\begin{array}{c}p=0.294 \\
X^{2}=2.445\end{array}$ \\
T/G & $32.7 \%(49)$ & $42.2 \%(43)$ & 0.143 & $0.67(0.4-1.12)$ \\
G/G & $6.7 \%(10)$ & $4.9 \%(5)$ & 0.787 & $1.39(0.46-4.18)$ \\
Total number of subjects & 150 & 102 & & \\
\hline
\end{tabular}

OR, odds ratio; $\mathrm{Cl}$, confidence interval. 
TABLE 6 | Significant association of MIR137 rs1625579 polymorphism with symptom expression degree according to PANSS in schizophrenia patients.

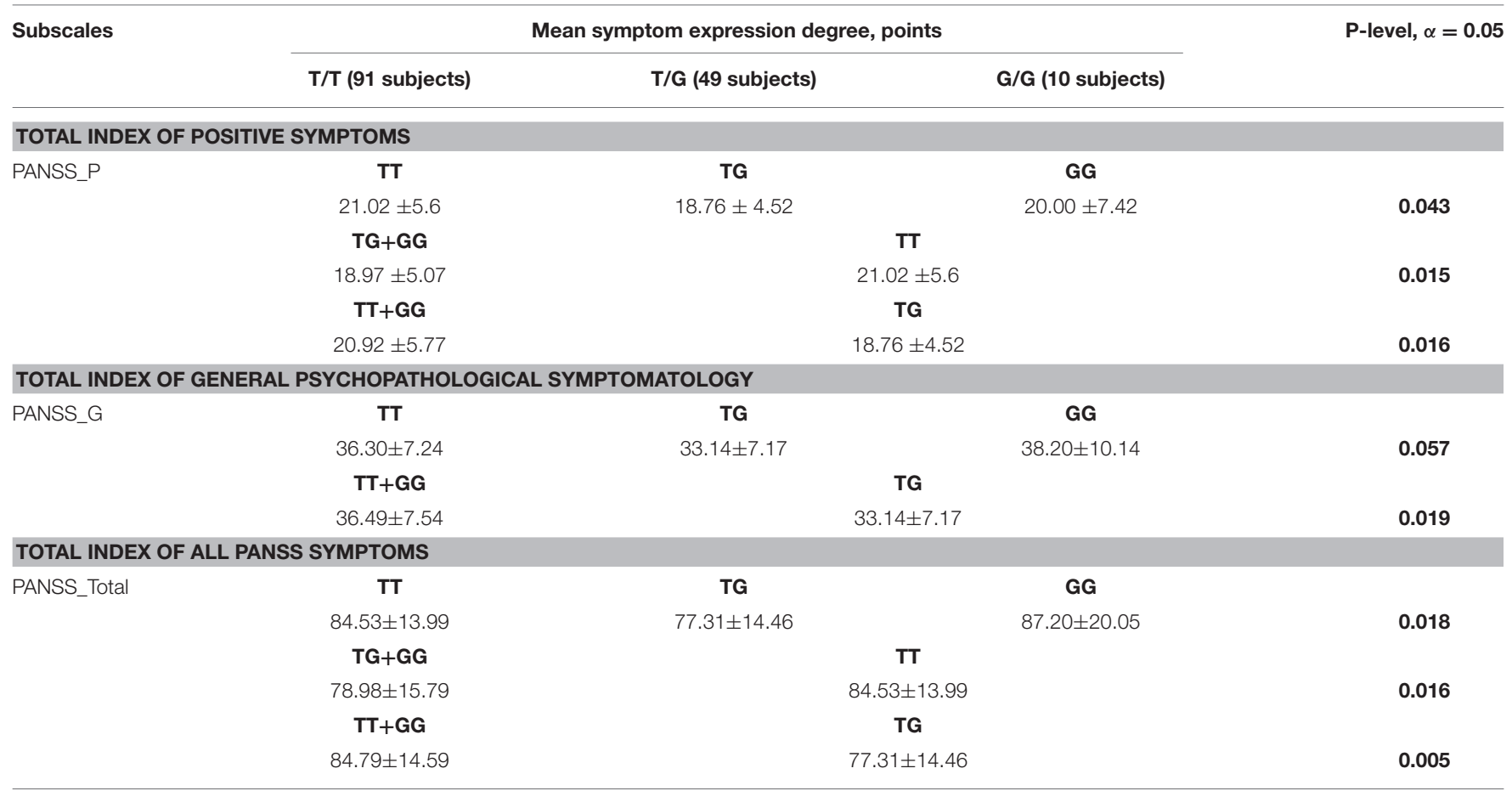

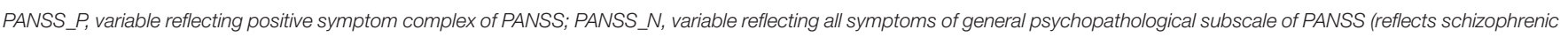

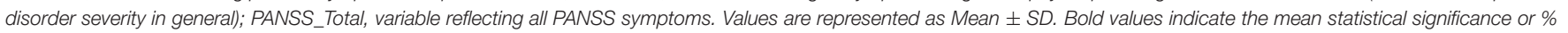
value.

severity in the studied sample seems to be difficult. Based on all, we can suppose that patients with $\mathrm{T} / \mathrm{T}$ and possibly G/G genotypes are characterized with somewhat more severely expressed symptomatology compared to heterozygous subjects $(p=0.005)$.

Another question that has arisen during our study was if the studied locus is related to the sex-dependent peculiarities of symptom expression. ANOVA analysis showed us the tendency in association of sex and rs1625579 MIR137 only for PANSS_Negative $(F=3.02, p=0.08)$. During the analysis of the association of rs 1625579 with symptom severity in men and women separately we found that female patients with $\mathrm{T} / \mathrm{T}$ genotype have significantly higher level of negative symptoms severity when compared to those with G-allele, as opposed to the male patient sample (Table 7). In male patients, the studied locus was significantly associated with one symptom only ("Poor attention") however it is very likely that this correlation might most probably appear false-positive because of the small sampling size of patients with G/G genotype. Positive symptoms and a number of general symptoms have not shown statistically significant association with sex separately, and this fact may be explained either with small sampling size or with sexual specificity. Moreover, we found that rs1625579 is associated with negative symptoms severity in female patients only, thus supposing certain sex-dependent differences exist in the modes of rs1625579 impact on pathophysiological processes of symptomatic pattern development. In the whole schizophrenia group, carriers of $(\mathrm{T} / \mathrm{G})$ genotype of MIR137
TABLE 7 | MIR137 rs1625579 polymorphism association with negative symptom expression degree according to PANSS in patients with schizophrenia.

\begin{tabular}{|c|c|c|c|}
\hline \multirow[t]{2}{*}{ Subscales } & \multicolumn{2}{|c|}{ Mean symptom expression degree, points } & \multirow{2}{*}{ P-level, $\alpha=0.05$} \\
\hline & $T / T$ & $\mathrm{~T} / \mathrm{G}+\mathrm{G} / \mathrm{G}$ & \\
\hline \multicolumn{4}{|c|}{ Female patients } \\
\hline & 54 subjects & 28 subjects & \\
\hline PANSS_N & $20.06 \pm 6.91$ & $17.00 \pm 5.87$ & 0.043 \\
\hline \multicolumn{4}{|c|}{ Male patients } \\
\hline & 38 subjects & 31 subjects & \\
\hline PANSS_N & $21.76 \pm 6.37$ & $22.55 \pm 8.16$ & 0.659 \\
\hline
\end{tabular}

Values are represented as Mean $\pm S D$. Bold values indicate the mean statistical significance or \% value.

rs1625579 polymorphism demonstrated less severe positive symptoms and general psychopathology.

Analysis of Wisconsin Card Sorting Test (WCST) data obtained from 129 patients with schizophrenia has not revealed any association of rs1625579 polymorphism with the studied parameters (Table 8).

\section{DISCUSSION}

The analysis of the published literature has demonstrated a very ambiguous pattern of MIR137 rs1625579 polymorphism 
TABLE 8 | MIR137 rs1625579 polymorphism association with WCST variables in scizophrenia patients.

\begin{tabular}{|c|c|c|c|}
\hline \multirow[t]{2}{*}{ WCST variable } & \multicolumn{3}{|c|}{ Genotypes } \\
\hline & $\begin{array}{c}\mathrm{T} / \mathrm{T} \\
\text { (83 subjects) }\end{array}$ & $\begin{array}{c}\text { T/G } \\
\text { (38 subjects) }\end{array}$ & $\begin{array}{c}\mathrm{G} / \mathrm{G} \\
\text { (8 subjects) }\end{array}$ \\
\hline Perseverative errors, \% & 17.8 & 19.1 & 16.4 \\
\hline Non-perseverative errors, \% & 28.3 & 32.3 & 16.0 \\
\hline Total of all errors, \% & 43.9 & 47.3 & 32.5 \\
\hline $\begin{array}{l}\text { Total amount of categories } \\
\text { completed (average } \\
\text { number) }\end{array}$ & 3.3 & 2.6 & 5.4 \\
\hline $\begin{array}{l}\text { Total amount of cards to } \\
\text { complete the first category } \\
\text { (average number) }\end{array}$ & 18.4 & 14.1 & 17.6 \\
\hline
\end{tabular}

associations with schizophrenia. According to the data presented in Table 1, three studies based on the GWAS that included Caucasian subjects have revealed a distinct association of rs1625579 locus with schizophrenia. However, a number of Chinese studies have not confirmed this association although it might be explained by the ethnical genetic differences. The Small sample size might also be a limiting factor for revealing the studied locus and schizophrenia association. In a recent meta-analysis on Chinese population studies which included 4 episode/control investigations with a total sample size of 2,847 patients and 3,018 healthy subjects, the authors have demonstrated significant association of the studied locus with schizophrenia for the following allelic models: $\mathrm{T}$ vs. G; T/T vs. $G / T+G / G$; T/T vs. G/G (21). On the other hand, another meta-analysis which included 11887 patients with schizophrenia and 16,660 healthy subjects has not revealed a studied locus and disease association in Asian populations, and it might be explained by the high heterogeneity in rs1625579 locus among continental populations (22).

Obtained data demonstrate high T-allele frequency of Belarusian indigenous population. T-allele is recognized as a major allele of MIR137 rs1625579 polymorphism for all known populations. The frequency of Genotypes distribution in the studied Belarusian indigenous population complied with the one known for the European populations (Electronic source: http://browser.1000genomes.org/Homo_sapiens/Variation/ Population? $\mathrm{db}=$ core; $\mathrm{r}=1: 98502434-98503434 ; \mathrm{v}=\mathrm{rs} 1625579$; $\mathrm{vdb}=$ variation; $\mathrm{vf}=1146801 \# 373401 \_$tablePanel). The studied polymorphism demonstrates frequency heterogeneity across the world populations, with similar frequencies observed (a) in East Asian and American populations, (b) in European and South Asian populations. East Asian populations possessed maximal percentage of $\mathrm{T} / \mathrm{T}$ genotype, as opposed to the European data. Since the world investigation data related to ambiguity of rs1625579 polymorphism and association with schizophrenia were mostly obtained from the East Asian populations, these ethnical differences were taken into account during the subsequent analysis and interpretation of this polymorphism association with schizophrenia risk.
Our analysis has revealed that MIR137 rs1625579 polymorphism is associated with positive symptoms severity as well as with schizophrenia severity in general (PANSS). It might be mediated by dysregulation of a number of significant signal pathways determining nervous system development and function. For instance, bioinformatic analysis carried out by Wright et al. (23) has demonstrated that 1,144 genes represent potential targets of this miRNA, while 25 of them are considered to be schizophrenia risk factors. MIR137 can influence on symptomatic indicators of patients and to interact with dopaminergic system. One of the miR-137 target genes is zinc finger protein $804 \mathrm{~A}(Z N F 804 A)(8,9)$. It participates in dopamine signaling pathways, and its target gene, catechol$O$-methyltransferase $(C O M T)$, can directly and selectively degrade dopamine in prefrontal cortex synapses through methylation (24). When COMT expression is inhibited, the reduced dopamine degradation in prefrontal cortex synapses causes dopamine hyperactivity that could lead to symptom manifestation (24). The other ZNF804A target gene associated with the dopamine pathway is the dopamine receptor D2 (DRD2) gene (24). When the dopaminergic system in the mesolimbic pathway is hyperactive, the hyperactivation of the postsynaptic membrane D2 receptor can induce positive symptoms, such as hallucinations and delusions (24).

Based on the data with sex differences in the locus rs1625579, it is possible to assume that MIR137 gene is involved in pathophysiological mechanisms leading to gender phenotypically distinct patterns of schizophrenia. Further investigation of the actual genes targeted by MIR137 and the relevant signal pathways might be promising for new locus-specific medicinal agents development. A similar analysis carried out in patients with schizophrenia from Singapore has demonstrated a significant increase $(p=0.016)$ in negative symptomatology in T/T patients compared to the subjects with $\mathrm{G} / \mathrm{G}$ or G/T genotypes (8). Data obtained by three independent Chinese investigation groups showed no association of the studied locus with scores for PANSS subscales $(14,16,25)$. Possibly, these controversial results may be explained by ethnical heterogeneity as well as the limited sample sizes.

According to WCST data, obtained association of MIR137 rs1625579 polymorphism with executive functioning have not been revealed but it could be false negative result due to small sample size. Kuswanto et al. (8) have analyzed rs1625579 polymorphism association with various neurocognitive domains, however, the significant association was revealed for executive functions only. This study has demonstrated that patients with schizophrenia homozygous by T allele have lower "attention" and "processing speed" cognitive variables. It's interesting that postmortem studies of brain tissue samples indicate that $\mathrm{T}$ allele may be associated with a decreased gene expression in dorsolateral region of the prefrontal cortex that is functionally involved into executive function in schizophrenia patients (10). Chinese research data published in 2014 have indicated that rs1625579 locus is associated with functional interaction between the dorsolateral region of the prefrontal cortex and hippocampal formation. Two hundred and ninety healthy subjects participated in this study. It was revealed that subjects homozygous by T-allele 
showed significant differences in abovementioned brain regions functional interactions compared to other genotypes. It was also demonstrated a strong association between the mentioned brain regions that predisposes to worse indices of working memory in $T / G$ but not in $T / T$ subjects. Therefore dorsolateral region of the prefrontal cortex is one of the most important brain regions involved in schizophrenia pathophysiology. Between this region and hippocamp connection formation, it was supposed that the studied polymorphism of MIR137 gene may influence schizophrenia pathophysiology by affecting these brain regions development and interaction plasticity (9). Research group from US has revealed that TT genotype is associated with left dorsolateral prefrontal cortex hyperactivation compared to G/G and G/T genotypes both in patients with schizophrenia and the control group (11). Another study focused on genes that expression was altered by variations in MIR137 expression. This list was cross-referenced with genome-wide schizophrenia association data to construct the individual polygenic scores. Increased polygenic risk within the empirically derived miR137 regulated gene score was associated with significantly lower performance in intelligence quotient test, worse working memory and episodic memory. These effects were observed most clearly at a polygenic threshold of $P=0.05$, although significant results were observed at all three thresholds analyzed. This result pointed out that MIR137 gene associated with the risk of schizophrenia might also be related to its broader downstream genetic effects (7). It's worthy of note that we failed to reveal any association of the rs1625579 MIR137 gene with Wisconsin Card Sorting Test (WCST) parameters of the number of cognitive processes that are predominantly controlled by prefrontal cortex. However, the data obtained in neurovisualization studies such as Functional Magnetic Resonance Imaging analysis etc. as well as studies based on different approaches to cognitive processes analysis have shown a functional significance of the mentioned locus for a number of cerebral structures, particularly prefrontal complex $(5,8)$.

In summary, the data obtained indicate that rs1625579 polymorphism of MIR137 gene is associated with symptom severity in patients with schizophrenia; furthermore, sexdependent differences of this association were revealed. The

\section{REFERENCES}

1. Issler O, Chen A. Determining the role of microRNAs in psychiatric disorders. Nat Rev Neurosci. (2015) 16:201-12. doi: 10.1038/nrn3879

2. Ha TY. MicroRNAs in human diseases: from autoimmune diseases to skin, psychiatric and neurodegenerative diseases. Immune Netw. (2011) 11:227-44. doi: 10.4110/in.2011.11.5.227

3. Davis GM, Haas MA, Pocock R. MicroRNAs: not "Fine-Tuners" but key regulators of neuronal development and function. Front Neurol. (2015) 6:245. doi: 10.3389/fneur.2015.00245

4. Schizophrenia Working Group of the Psychiatric Genomics Consortium: Ripke S, Neale BM, Corvin A, Walters JT, Farh KH, Holmans PA, et al. Biological insights from 108 schizophrenia-associated genetic loci. Nature (2014) 511:421-27. doi: 10.1038/nature13595

5. Hommers LG, Domschke K, Deckert J. Heterogeneity and individuality: microRNAs in mental disorders. J Neural Transm. (2015) 122:79-97. doi: $10.1007 /$ s00702-014-1338-4 highest severity of symptoms was observed in patients carrying $\mathrm{T} / \mathrm{T}$ genotype that is consistent with data on $\mathrm{T} / \mathrm{T}$ genotype pathogenicity for nervous system functioning. Given the fact that a reduced adjusted incidence of cancer was noted in patients suffering from schizophrenia (by potential mechanisms such as excess of dopamine, enhanced natural killer cell activity, increased apoptosis, modulated cytochrome enzymes by antipsychotics that block mutagen activation, or vitamin $\mathrm{D}$ deficiency) (26) and miR-137 is considered as a cancer therapeutic target (27), our study could explain the lower incidence of cancer in schizophrenia compared to the general population. Taking into account high significance of MIR137 for nervous system development and function, it's necessary to emphasize the importance of complex methodological approach to the studies of gene-phenotype interactions focusing on cognitive processes, that represent the basis of schizophrenia pathophysiology.

\section{AUTHOR CONTRIBUTIONS}

HK, AN, IG, ND, AM, VO, OD, and NW designed the study and supervised the draft completion. HK, IG, ND, AM, and OD did genetic analyzes. $\mathrm{HK}, \mathrm{AN}$, and $\mathrm{VO}$ collected the cognitive and clinical data, managed the literature searches and performed the data analyses. HK, AN, and NW wrote the first draft of the manuscript. All authors contributed to and approved the final draft of the manuscript.

\section{FUNDING}

This study was financially supported by the research grant of Belarusian Republican Foundation of Fundamental Research DNA methylation system gene polymorphism association with schizophrenia clinical variants Belarusian (N M13M-065/16th Apr, 2013).

\section{ACKNOWLEDGMENTS}

We thank the subjects who took part in this study.

6. Warburton A, Breen G, Bubb VJ, Quinn JP. A GWAS SNP for schizophrenia is linked to the internal MIR137 promoter and supports differential allele-specific expression. Schizophr Bull. (2016) 42:1003-8. doi: $10.1093 /$ schbul/sbv144

7. Cosgrove D, Harold D, Mothersill O, Anney R, Hill MJ. MiR-137derived polygenic risk: effects on cognitive performance in patients with schizophrenia and controls. Transl Psychiatry (2017) 7:e1012. doi: 10.1038/tp.2016.286

8. Kuswanto CN, Sum MY, Qiu A, Sitoh YY, Liu J, Sim K. The impact of genome wide supported microRNA-137 (MIR137) risk variants on frontal and striatal white matter integrity, neurocognitive functioning, and negative symptoms in schizophrenia. Am J Med Genet B Neuropsychiatr Genet. (2015) 168B:317-26. doi: 10.1002/ajmg.b.32314

9. Liu B, Zhang X, Hou B, Li J, Qiu C, Qin W, et al. The impact of MIR137 on dorsolateral prefrontal-hippocampal functional connectivity in healthy subjects. Neuropsychopharmacology (2014) 39:2153-60. doi: $10.1038 /$ npp.2014.63 
10. Guan F, Zhang B, Yan T, Li L, Liu F, Li T, et al. MIR137 gene and target gene CACNA1C of miR-137 contribute to schizophrenia susceptibility in Han Chinese. Schizophr Res. (2014) 152:97-104. doi: 10.1016/j.schres.2013. 11.004

11. Van Erp TG, Guella I, Vawter MP, Turner J, Brown GG, McCarthy G, et al. Schizophrenia miR-137 locus risk genotype is associated with dorsolateral prefrontal cortex hyperactivation. Biol Psychiatry (2014) 75:398-405. doi: 10.1016/j.biopsych.2013. 06.016

12. Patel VS, Kelly S, Wright C, Gupta CN, Arias-Vasquez A, PerroneBizzozero N, et al. MIR137HG risk variant rs1625579 genotype is related to corpus callosum volume in schizophrenia. Neurosci Lett. (2015) 602:44-9. doi: 10.1016/j.neulet.2015.06.039

13. $\mathrm{Li} \mathrm{M}, \mathrm{Su} \mathrm{B}$. Impact of the genome-wide schizophrenia risk single nucleotide polymorphism (rs1625579) in miR-137 on brain structures in healthy individuals. Psychiatr Genet. (2013) 23:267. doi: 10.1097/YPG.0000000000000011

14. Sun YJ, Yu Y, Zhu GC, Sun ZH, Xu J, Cao JH, et al. Association between single nucleotide polymorphisms in MiR219-1 and MiR137 and susceptibility to schizophrenia in a Chinese population. FEBS Open Bio. (2015) 5:774-8. doi: 10.1016/j.fob.2015.08.008

15. Yuan J, Cheng Z, Zhang, F, Zhou Z, Yu S, Jin C. Lack of association between microRNA-137 SNP rs1625579 and schizophrenia in a replication study of Han Chinese. Mol. Genet. Genomics (2015) 290:297-301. doi: 10.1007/s00438-014-0924-3

16. Wang S, Li W, Zhang H, Wang X, Yang G, Zhao J, et al. Association of microRNA137 gene polymorphisms with age at onset and positive symptoms of schizophrenia in a Han Chinese population. Int J Psychiatry Med. (2014) 47:153-68. doi: 10.2190/PM.47.2.f

17. Nestsiarovich A, Obyedkov V, Kandratsenka H, Siniauskaya M, Goloenko I, Waszkiewicz N. Disorganization at the stage of schizophrenia clinical outcome: Clinical-biological study. Eur Psychiatry (2017) 42:44-8. doi: 10.1016/j.eurpsy.2016.12.011

18. Kay SR, Fiszbein A, Opler LA. The positive and negative syndrome scale (PANSS) for schizophrenia. Schizophr Bull. (1987) 13:261-76. doi: $10.1093 /$ schbul/13.2.261

19. Wiłkość M, Szałkowska A, Skibinska M, Zajac-Lamparska L, Maciukiewicz $\mathrm{M}$, Araszkiewicz A. BDNF gene polymorphisms and haplotypes in relation to cognitive performance in Polish healthy subjects. Acta Neurobiol Exp. (2016) 76:43-52. doi: 10.21307/ane-2017-004

20. Mathew CG. The isolation of high molecular weight eukaryotic DNA. Methods Mol Biol. (1985) 2:31-4. doi: 10.1385/0-89603-064-4:31

21. Zhang P, Bian Y, Liu N, Tang Y, Pan C, Hu Y, et al. The SNP rs1625579 in miR-137 gene and risk of schizophrenia in Chinese population: a metaanalysis. Compr Psychiatry (2016) 67:26-32. doi: 10.1016/j.comppsych.2016. 02.009

22. $\mathrm{Pu} \mathrm{X}$, Xiao X. No evidence of an association between MIR137 rs1625579 and schizophrenia in Asians: a meta-analysis in 30843 individuals. Psychiatr Genet. (2016) 26:203-10. doi: 10.1097/YPG.00000000000 00136

23. Wright C, Turner JA, Calhoun VD, Perrone-Bizzozero N. Potential impact of miR-137 and its targets in schizophrenia. Front Genet. (2013) 4:58. doi: 10.3389/fgene.2013.00058

24. Yin J, Lin J, Luo X, Chen Y, Li Z, Ma G, et al. miR-137: a new player in schizophrenia. Int J Mol Sci. (2014) 15:3262-71. doi: 10.3390/ijms15023262

25. Ma G, Yin J, Fu J, Luo X, Zhou H, Tao H, et al. Association of a miRNA137 polymorphism with schizophrenia in a Southern Chinese Han population. Biomed Res Int. (2014) 2014:751267. doi: 10.1155/2014/751267

26. Mahmoudi E, Cairns MJ. MiR-137: an important player in neural development and neoplastic transformation. Mol Psychiatry (2017) 1:44-55. doi: $10.1038 / \mathrm{mp} .2016 .150$

27. Kisely S, Crowe E, Lawrence D. Cancer-related mortality in people with mental illness. JAMA Psychiatry (2013) 2:209-17. doi: 10.1001/jamapsychiatry.2013.278

Conflict of Interest Statement: The authors declare that the research was conducted in the absence of any commercial or financial relationships that could be construed as a potential conflict of interest.

Copyright (C) 2018 Kandratsenka, Nestsiarovich, Goloenko, Danilenko, Makarevich, Obyedkov, Davydenko and Waszkiewicz. This is an open-access article distributed under the terms of the Creative Commons Attribution License (CC BY). The use, distribution or reproduction in other forums is permitted, provided the original author(s) and the copyright owner(s) are credited and that the original publication in this journal is cited, in accordance with accepted academic practice. No use, distribution or reproduction is permitted which does not comply with these terms. 